\title{
A review on the importance of genotyping and phenotyping in fluoropyrimidine treatment
}

\author{
Andrada-Larisa Deac ${ }^{1}$, Claudia Cristina Burz ${ }^{1,2}$, Horea Florin Bocșe ${ }^{3}$, \\ Ioana Corina Bocșan ${ }^{4}$, Anca-Dana Buzoianu ${ }^{4}$
}

1) "Prof. Dr. Ion Chiricuţă" Oncology Institute, Cluj-Napoca, Romania

2) Immunology and Allergology, Department 2 - Functional Sciences, Faculty of Medicine, Iuliu Hatieganu University of Medicine and Pharmacy, Cluj-Napoca, Romania

3) "Octavian Fodor" Regional Institute of Gastroenterology and Hepatology, $3^{\text {rd }}$ General Surgery Clinic, Cluj-Napoca, Romania

4) Pharmacology, Toxicology and Clinical Pharmacology, Department 2 - Functional Biosciences, Faculty of Medicine, Iuliu Hatieganu University of Medicine and Pharmacy, ClujNapoca, Romania

DOI: $10.15386 / \mathrm{mpr}-1564$

Manuscript received: 04.01.2020

Received in revised form: 24.02 .2020

Accepted: 20.03.2019

Address for correspondence:

andrada.deac@gmail.com

This work is licensed under a Creative Commons Attribution-NonCommercialNoDerivatives 4.0 International License

\begin{abstract}
Fluoropyrimidines, after more than 50 years from their discovery, are still the treatment of many types of cancer, and it is estimated that two million patients receive fluoropyrimidine therapy annually. The toxicity associated with fluoropyrimidines affects $30-40 \%$ of patients and some adverse effects can be lethal.

Dihydroypyrimidine dehydrogenase is the main enzyme in the catabolism of 5-FU and DPD activity deficiency can cause important toxicity. This is an important reason to determine DPD activity in order to improve patient safety and to limit potential life-threating toxicity.

At presentmultiple phenotypic and genotypic methods are available for the determination of DPD activity, some of these methods have proven their usefulness in practice, and yet they are not routinely recommended in clinical practice.

This review is another statement of the importance of the determination of DPD status, the phenotypic and genotypic methods that are available and can be used.
\end{abstract}

Keywords: chemotherapy, fluoropyrimidine treatment, toxicity

\section{Introduction}

Fluoropyrimidines still represent the foundation stone in the treatment of multiple cancers, such as colorectal cancer, gastric cancer, pancreatic cancer, esophageal cancer, head and neck cancer, breast cancer and every year it is estimated that two millions patients are treated with fluoropyrimidine $[1,2]$. The fluoropyrimidines used in general practice are 5-Fluorouracil (5-FU), Capecitabine and Tegafur, the last two are prodrugs of 5-Fluorouracil.

Capecitabine, the oral precursor of 5-Fluorouracil, is administered in advanced colorectal cancer, gastric cancer, and breast cancer, turning into 5-Fluorouracil at the tumor site $[3,4,5]$. Capecitabine passes unchanged through the intestinal wall and is converted in the liver to 5-deoxy-5-fluorocitidine (5'$\mathrm{dFCR}$ ) and then to 5-deoxy-fluorouridine (5'-dFUR), by carboxylesterase and cytidine-deaminase. 5'-dFUR turns into 5 -FU by thymidine-phosphorylase or uridinephosphorylase [6,7].

Tegafur, another prodrug of
5-Fluorouracil, is converted by CYP2A6 to an intermediate metabolite, 5- hydroxytegafur, which spontaneously breaks down to $5-\mathrm{FU}[6,7]$.

\section{5-Fluorouracil: history, metabolic pathways and toxicity}

5-Fluorouracil was patented in 1956 and become used in cancer treatment in 1962. In 1985, Tuchman et al. reported the first case of severe toxicity in a patient with familial pyridinemia and treatment with 5-FU. This was the first evidence that a genetic defect in pyrimidine catabolism can be associated with fluoropyrimidine-related toxicity. Now, we know that DPD activity is associated with 5-FU pharmacology and enzyme deficiency increases the risk of severe side effects, sometimes even lethal, in patients undergoing treatment with fluoropyrimidines [1-4].

In Japan sixteen deaths were reported after simultaneous administration of 5-FU and Sorivudine, which inhibits the activity of DPD. Sorivudine is catabolized in the intestinal wall to uracil, which inhibits dihydropyrimidine dehydrogenase 
(DPD), thus resulting into a prolonged systemic exposure to 5 -FU $[2,3]$.

After administration of $5-\mathrm{FU}$, only $1-5 \%$ of the amount is transformed at the intracellular level into cytotoxic metabolites such as fluorodeoxyuridine-monophosphate (FdUMP), fluorodeoxyuridine- diphosphate (FdUDP), fluorodeoxyuridine triphosphate (FdUTP) and fluorouridine triphosphate (FUTP). The main anti-tumoral mechanism of 5-FU is due to the cytotoxic metabolite FdUMP, which inhibits thymidylate-synthase (TS), a crucial enzyme in the DNA replication and repair. TS is an important enzyme for de novo synthesis of thymidylate required for DNA synthesis. FdUMP and FdUTP destroy the processes and function of DNA, by inhibition of DNA elongation and fragmentation, respectively FUTP influences the RNA processes of methylation, stability and synthesis [1-7].

About $80-85 \%$ of 5 -FU is catabolized by dihydropyrimidine dehydrogenase (DPD) into the non-cytotoxic metabolite, 5-fluoro-5,6-dihydrouracil (DHFU) [7]. DHFU is then converted to fluoro-betaureidopropionate (FUPA) and fluoro-beta-alanine (FUBA), which are eliminated from the body through urine [7]. So
DPD is the main responsible enzyme for the catabolism of 5-FU. Because DPD is the first enzyme in the 5-FU catabolism, the amount of 5-FU which is converted into cytotoxic metabolites depends on the systemic activity of DPD [6,7] (Figure 1).

5-FU has a narrow therapeutic window so there is a small limitation between efficacy and the maximum tolerated dose. A decreased DPD activity means a decreased clearance and an increased half-life of 5- FU resulting an increased risk of dose-related toxicity $[7,8]$.

Dihydropyrimidine dehydrogenase (DPD) is the most important enzyme for fluoropyrimidine catabolism pathway. Patients with a partial or complete DPD deficiency are at risk to develop severe toxicity due to the treatment with fluoropyrimidines [8,9]. DPD activity can be determined in many tissues, but the most intense activity is found in the liver, where catabolism occurs. Patients with decreased hepatic function are candidates for developing severe toxicity after treatment with fluoropyrimidines. DPD determination from the normal liver correlates with DPD activity from the peripheral blood mononuclear cells (PBM), so PBM level of DPD is equivalent to the total amount of DPD [8,9].

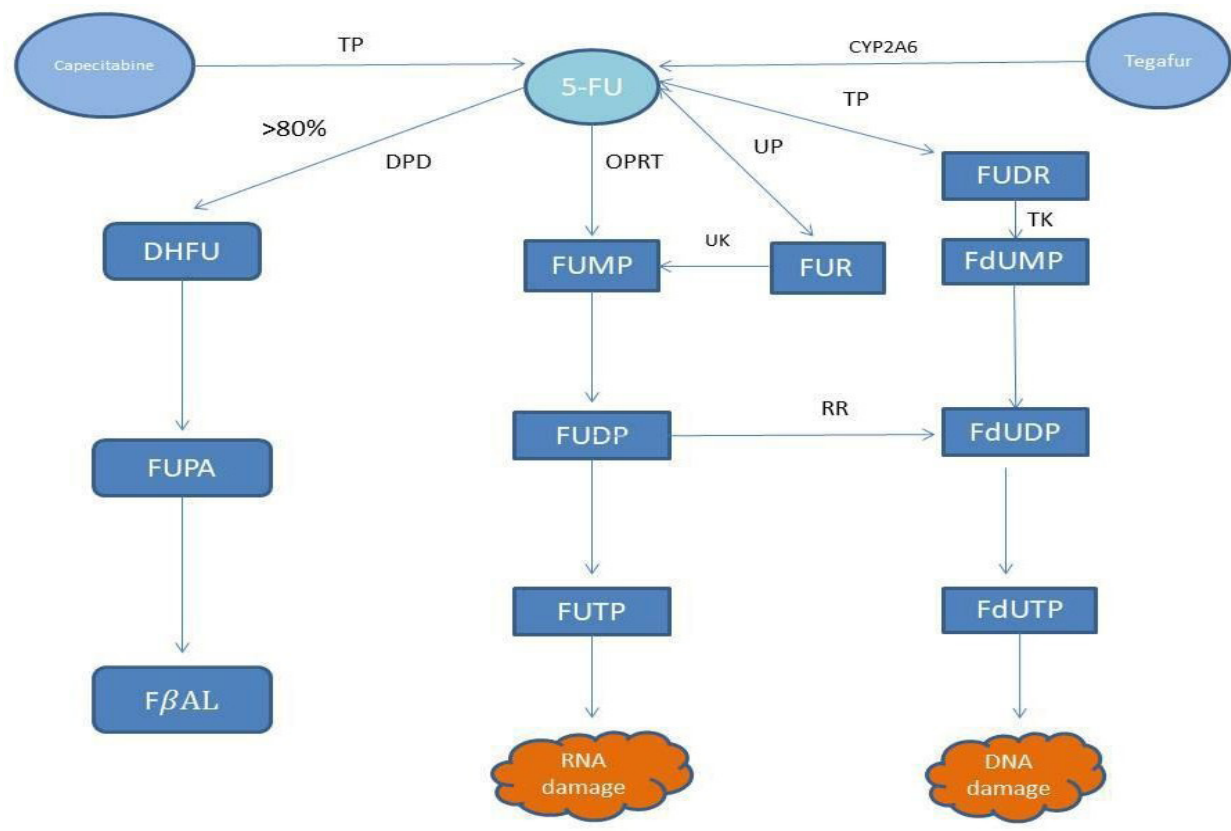

Figure 1. 5-FU metabolic pathways. Capecitabine and Tegafur are converted to 5-FU by thymidine-phosphorylase (TP) and CYP2A6. $1-5 \%$ of $5-\mathrm{FU}$ is transformed into cytotoxic metabolites fluorodeoxyuridine-monophosphate (FdUMP), fluorodeoxyuridine-diphosphate (FdUDP), fluorodeoxyuridine triphosphate (FdUTP) and fluorouridine triphosphate (FUTP). 5-FU is directly transformed to fluoridine monophosphate (FUMP) by orotate phosphoribosyltransferase (OPRT), or indirectly through fluorouridine (FUR) by uridine phosphorylase (UP) and uridine kinase (UK). FUMP is then phosphorylated to fluorouridine diphosphate (FUDP), which is phosphorylated to fluorouridine triphosphate (FUTP). FUDP can also by converted to fluorodeoxyuridine diphosphate (FdUDP) by ribonucleotide reductase (RR). In an alternative activation pathway 5-FU is converted to fluorodeoxyuridine (FUDR) by thymidine phosphorylase. FUDR is the converted through thymidine phosphate in fluorodeoxyridine monophosphate (FdUMP), which is phosphorylated to fluorodeoxyuridine diphosphate (FdUDP), which is phosphorylated to fluorodeoxyuridine triphosphate (FdUTP). $80 \%$ of the 5-FU is catabolized by dihydropyrimidine dehydrogenase into the non-cytotoxic metabolites 5-fluoro-5,6-dihydrouracil (DHFU), which is then converted to fluoro-beta-ureidopropionate (FUPA) and fluoro-beta-alanine (FUBA), which are eliminated through urine. 
Bosidron-Celle et al. compared the 5-FU clearance in two heterozygote patients with DPYD*2A and seven heterozygote patients with DPYD*9B with 163 patients with no deficient variant. In the heterozygote patients with the two deficient variants they observed that 5-FU clearance had decreased by $80 \%$ and $40-58 \%$ respectively, compared to the patients with no deficient variant [8]. The same group found in one patient three heterozygote variants: DPYD*2A, rs67376798 and $85 \mathrm{~T}>\mathrm{C}$ resulting in a 5-FU plasma clearance close to zero. All these data indicate that heterozygote patients for deficient variants have significantly reduced fluorouracil clearance, by $40-80 \%$, compared to patients with no deficient variants [6-10].

DPD activity turned out to be inversely proportional with plasma concentration of 5-FU in patients treated with continuous infusion [8-10]. A study showed a concentration area under the curve of 5-FU increased from 9.2 in group control to 24.7 in patients with DPD deficiency [10].

Fluoropyrimidines induce grade 3-4 toxicity in 30$40 \%$ of cases and lethal toxicity in $0.3-2 \%$ of cases (almost $5 \%$ of the elderly population). The level of DPD activity has an important inter-individual variability which is partly explained through different variants of DPYD gene which encodes DPD [11-13].

The complete deficit can be very rare, $0.1-0.5 \%$ in the general population and partial deficiency can be found in $3-10 \%$ in Caucasian population. DPD deficiency can be detected in $39-61 \%$ of patients with severe toxicity [14].

Strategies to determinate the DPD deficiency are based on genotyping and phenotyping. The main phenotyping methods are measuring DPD enzyme activity in PBM, determination of the endogenous concentration of uracil and dihydrouracil in plasma, saliva and urine, $2-{ }^{13} \mathrm{C}$ uracil breath test, uracil dose test $[15,16]$.

\section{Phenotyping methods - what to choose from so many alternatives?}

Measuring DPD activity in PBM is the gold standard in phenotyping methods, the level of PBM activity correlates with the hepatic level. The clinical validity in measuring DPD enzyme activity in PBM has been demonstrated in many retrospective and prospective studies [15,16]. An important point of view is the DPD activity threshold value, which indicates whether there is a deficit or not. Milano et al. have proposed a cut-off under $70 \%$ of the population mean to classify patients with enzyme deficiency [14]. This value has been subsequently validated as a predictive value for fluoropyrimidine related toxicity in many retrospective studies.

An important phenotyping method to determinate the interindividual variation in DPD activity is the $2{ }^{13} \mathrm{C}$ uracil breath test, an oral aqueous solution of $2-{ }^{13} \mathrm{C}$ uracil which decomposes into ${ }^{13} \mathrm{CO}_{2}$ and can be detected from respiration by infrared spectroscopy [16,17]. Mattison et al. demonstrated in a study on 58 patients, 50 with normal
DPD activity, 7 with partial deficiency and one with total deficiency, who were submitted to this test at different time intervals up to 180 minutes, that measuring the level of $2-{ }^{13} \mathrm{C}$ uracil at 50 minutes may detect the deficiency [16]. Another study showed that $2-{ }^{13} \mathrm{C}$ uracil breath test has a moderate accuracy to differentiate between individuals with severe toxicity and moderate toxicity or not at all [17]. An important limitation of this test is the fact that the obtained results can be related to other enzymes from 5-FU metabolism, such as dihydropyrimidase and betaureidopropionase $[18,19]$.

In the uracil dose test an oral solution of uracil is administrated to the patient and then dihydrouracil and uracil pharmacokinetics are measured from plasma at different time intervals. van Staveren el al. compared the pharmacokinetics in two groups with comparable characteristics, except age, DPD status, and disease [20]. The group with normal DPD activity was established from young healthy individuals, and the group with DPD deficiency were patients with colorectal and breast cancer. Age is a factor that influences pharmacokinetics by functional modifications that come with age, therefore aging decreases hepatic perfusion and therefore we have a decreased clearance for some drugs through the first liver passage. Also, the presence of hepatic metastases and induced chemotherapy steatosis decrease the metabolism of drugs, which can lead to the alteration in the metabolism of uracil [20].

The pretreatment endogenous concentration of dihydrouracil and uracil from plasma has been investigated for determination of enzymatic activity of DPD. The association between UH2/Ura ratio, 5-FU clearance and treatment associated toxicity was demonstrated. In one study which enrolled 66 patients diagnosed with colorectal cancer, the mean value of $\mathrm{UH} 2 / \mathrm{Ura}$ ratio in patients with adverse effects has statistically significantly lower (3.2 versus $7.1, p=0.009$ ) than the reference population, healthy population. Twenty-one from twenty-four patients which presented adverse effects had a cut- off under 4, determined by ROC curve. UH2/Ura ratio has predictive for fluoropyrimidine related toxicity with a $\mathrm{Se}=87 \%$ and $\mathrm{Sp}=93 \%[16-19]$.

Carlsson et al. have determined pre-treatment dihydrouracil and uracil from the saliva of patients with colorectal cancer [23]. They took samples of saliva in fasting conditions from 73 patients before starting the adjuvant treatment, and dihydrouracil and uracil were measured by high-performance liquid chromatography (HPLC). The adverse effects were monitored before and after every cycle of chemotherapy and the most severe adverse reactions were noted. From the 73 patients, 56 patients needed a dose reduction due to side effects and 8 had to interrupt the treatment due to severe toxicity. The $\mathrm{UH} 2 /$ Ura ratio was low in these patients who experienced toxicity due to treatment with fluoropyrimidines [20-23]. 
The same group of authors determined the dihydrouracil and uracil from urine levels from 143 patients with colorectal cancer and compared them to the levels of control group [23]. The urine samples were taken from the first urine in the morning and measured with HPLC. According to this study, there was no statistically significant evidence between patient group and control group. In another study, Hayashi el al. established a threshold value for uracil and dihydrouracil in Japanese adult patients. The mean SD dihydrouracil and uracil levels were found to be 63.8-66.0 $1 \mathrm{~mol} / \mathrm{g}$ and $23.8-35.5$ $1 \mathrm{~mol} / \mathrm{g}$ creatinine, and the mean SD UH2/Ura ratio was $0.36-0.1$; these values are much lower compared to the values recorded by Carlsson el al [23-25].

Kristensen et al. determined the UH2/Ura ratio in plasma at 68 patients with colorectal cancer and 100 healthy individuals who formed the group control. Twenty-four patients with adverse effects were tested for DPYD*2A, and UH2/Ura ratio was low. Plasma concentration of 5-FU increased 130 times in 13 of the 24 patients [15].

Meulendijks et al. in a study published in 2017, show that pretreatment serum uracil was superior to dihydrouracil/uracil ratio as a predictor for severe toxicity associated with fluoropyrimidine treatment [26]. Serum uracil was strongly predictive of global toxicity $(p=0.004)$, gastrointestinal toxicity $(p=0.0006)$, toxicity-related hospitalization $(\mathrm{p}=0.011)$ and fatal toxicity $(\mathrm{p}=0.007)$, but not associated with hematological toxicity $(\mathrm{p}=0.383)$. The pretreatment uracil concentration ranged between 3.2$38.2 \mathrm{ngml}^{-1}$, and patients were classified in groups based on the distribution of concentration, $U>16 \mathrm{ngml}^{-1}, 13.9$ $16 \mathrm{ngml}^{-1}, 13-13.9 \mathrm{ngml}^{-1}$ and $U<13 \mathrm{ngml}^{-1}$. The first two groups, Uracil $>16 \mathrm{ngml}^{-1}$ and $13.9-16 \mathrm{ngml}^{-1}$ were at statistically significant increased risk of global toxicity compared with the group $<13 \mathrm{ngml}^{-1}$, with $\mathrm{OR}=5.3$ and $\mathrm{p}=0.0087$, respectively $\mathrm{OR}=8.2$ and $\mathrm{p}=0.0004$. Patients with pretreatment concentration of 13-13.8 $\mathrm{ngml}^{-1}$, had no statistically significant risk [26-28].

\section{utility \\ Genotyping, yet another proof of clinical}

The most important methods are those of genotyping for different genetic defects, such as the presence of a deleterious mutation in DPYD and mutation in miR-27A which influence the expression of DPD, but for which only a few pieces of information exist. The most studied is the DPYD gene that encodes DPD activity. The toxicity of 5-FU can be influenced by other genes, the most studied are ABCB1, MTHFR, and TYMS, but data are inconsistent and strategies to determine the activity of these genes are needed [29,30]. TYMS variants located in the regulatory regions seem to have an influence in the transcription rate. High intra-tumoral levels of TYMS have been associated with 5-FU resistance [29,30].

In terms of MTHFR, two single nucleotide polymorphism (SNPs) located on exon 4 and exon 7 seem to lead to a decreased enzymatic activity according to some in vitro studies [30].

DPYD gene is located on chromosome $1 \mathrm{p} 22$ and contains 4399 nucleotides in 23 exons which encode 1025 amino acids such as uracil, flavine mononucleotide (FMN), NADPH and flavine adenine dinucleotide (FAD) binding sites, along with four Sulphur motifs [31-33].

The most frequent DPYD allelic variants are DPYD*5 (rs1801159 T>C), DPYD*6 (rs1801160 C >T), and DPYD*9A ( $r 1801265 \mathrm{~A}>\mathrm{G})$ at frequencies of 11.5$30,0.7-9$, and $2.9-13.7 \%$, respectively, and data regarding their effects on DPD activity are contradictory. The Dutch pharmacogenetics working group have designated these variants as functional on the lack of an association between toxicity and these variants [33,34].

The most studied variant is DPYD*2A (IVS14 + $1 \mathrm{G}>\mathrm{A}$, c. $1905+1 \mathrm{G}>\mathrm{A}$, or rs3918290) which is a single nucleotide polymorphism (SNP), at the intron boundary of exon 14 and result in a splicing defect, skipping of the entire exon and resulting in a non-functional protein. This allele is considered to be more common than others variants at a frequency range from $0.004-3.5 \%[33,34]$.

DPYD*2A was associated with multiple lethal events during the treatment with fluoropyrimidines. For example in a paper published in 2001 by van Kuilenburg et al., one female patient died because of adverse effects of the treatment, she presented stomatitis after the first administration of the drug, and after the second cycle of chemotherapy fever, severe stomatitis, leucopenia, thrombocytopenia, pancytopenia and died after the infection complications. Genotyping found that she had DPYD*2A, homozygous [29].

DPYD*2A was correlated statistically significantly with nausea and vomiting $(\mathrm{p}<0.007)$ and neutropenia $(\mathrm{p}<0.001)$, DPYD*9B with dehydration $(\mathrm{p}=0.02)$, diarrhea $(p=0.003)$, leucopenia $(p=0.002)$, neutropenia $(p<0.001)$ and thrombocytopenia $(\mathrm{p}<0.001)$ [34].

In a meta-analysis, Terrazzino et al. showed that DPYD*2A variant is a risk factor of grade 3 or higher hematological toxicity and to a less grade 3 or higher mucositis or diarrhea [34].

DPYD*3 (rs72549303 C >del), ${ }^{*} 13$ (rs55886062 $\mathrm{A}>\mathrm{C}$ ), and $* 9 \mathrm{~B}$ (rs67376798) are relatively rare but result in low DPD activity and 36,37 seems to be related to 5-FU toxicity [33,34]. Recently in African-American patients, a novel DPYD variant, Y186C, was identified and it seems that it can be found in $26 \%$ of patients with reduced DPD activity. Individuals carrying this variant had a $46 \%$ reduction in enzyme activity as compared with noncarriers [33]. 
Table I. DPYD variants and dose reduction recommendations.

\begin{tabular}{|c|c|c|c|c|c|c|}
\hline $\begin{array}{l}\text { DPYD } \\
\text { variant }\end{array}$ & $\begin{array}{l}\text { NCBI SNP } \\
\text { reference }\end{array}$ & Exon & Nucleotide & Protein & MAF & $\begin{array}{l}\text { Dosing recommendation } \\
\text { heterozygous/homozygous }\end{array}$ \\
\hline $\begin{array}{l}\text { DPYD*2A } \\
(\text { IVS } 14+1 G>A \text {, } \\
\text { c. } 1905+1 G>A)\end{array}$ & rs3918290 & $\begin{array}{c}\text { Intron } \\
14\end{array}$ & IVS14+1G $>A$ & $\begin{array}{l}\text { Exon } 14 \\
\text { skipping }\end{array}$ & $3.5 \%$ & $50 \% /$ Contraindicate \\
\hline $\begin{array}{l}\text { DPYD*9B } \\
(c .2846 A>T)\end{array}$ & rs67376798 & 22 & A2846T & D949V & $0.1-1.1 \%$ & $25 \% / 50 \%$ \\
\hline $\begin{array}{l}\text { DPYD*13 } \\
(\text { c. } 1679 \mathrm{~T}>\mathrm{G})\end{array}$ & rs55886062 & 13 & $\mathrm{~T} 1679 \mathrm{G}$ & I560S & $0-2 \%$ & $25 \%-50 \%$ \\
\hline $\begin{array}{l}\text { HapB3 (c.1129- } \\
5923 \mathrm{C}>\mathrm{G})\end{array}$ & rs75017182 & 1 & & $\mathrm{E} 412 \mathrm{E}$ & & $25 \% / 50 \%$ \\
\hline
\end{tabular}

Abbreviations: DPYD - the gene that encodes DPD, HapB3 - haplotype B3, MAF - minor allele frequency, NCBI - National Center for Biotechnology Information

Another well studied variant is DPYD*9B, c. $2846 \mathrm{~A}>\mathrm{T}$, described for the first time in 2000 by van Kuilenberg et al [34-36]. The c.2846A $>\mathrm{T}$ polymorphism leads to a structural change in the enzyme that interferes with a cofactor binding or electron transport. The frequency of this allele is between $0.1-1.1 \%$. In vitro studies show that homozygous patients with c. $2846 \mathrm{~A}>\mathrm{T}$ variant have a $60 \%$ activity of the enzyme compared with wild-type [35,36]. Therefore homozygous patients of this variant need a $50 \%$ reduction of the dose and heterozygous patients a reduction of $25 \%$ [36]. Rosmarin et al. in a study which enrolled 927 patients evaluated the toxicity induced by Capecitabine and found that DPYD*9B variant and DPYD*2A variant is the most frequent variants found in a relationship with Capecitabine toxicity, $\mathrm{OR}=5.51, \mathrm{p}=0.0013$ [30].

The B3 haplotype, a combination of various genetic changes, corresponds to an allele with several variations in linkage disequilibrium (c.1236G $>A$ ) in exon 1 a four intronic variations C. $483+18 \mathrm{G}>\mathrm{A}$, c. $680+139 \mathrm{G}>\mathrm{A}$, c. 959 $51 \mathrm{~T}>\mathrm{G}$, and c.1129-5923C $>\mathrm{G}$ ). The c.1129-5923C $>\mathrm{G}$ affects pre-mRNA splicing and probably represents the causal variation of Hap B3 [36]. Homozygous patients with variant c. $1236 \mathrm{G}>\mathrm{A} / \mathrm{Hap} \mathrm{B} 3$ have a $50 \%$ decrease in DPD activity. These data support a dose reduction of $25 \%$ in patients with heterozygous form and $50 \%$ in homozygous patients [36].

A meta-analysis which enrolled 7365 patients from 8 studies highlighted the fact that DPYD*13 and c1236G $>$ A/ Hap B3 are statistically significantly associated with high risk for fluoropyrimidines related- toxicity [36].

In a study including 2886 patients with stage III colorectal cancer, 2594 patients developed side effects, they were genotyped for DPYD*2A, DPYD*9B and DPYD*13. The incidence of grade 3 or greater adverse effects was $88 \%$ for DPYD*2A, $81.5 \%$ for DPYD*9B and $50 \%$ for DPYD*13. Statistically significant associations were demonstrated between grade 3 or greater 5-FU adverse effects for both DPYD*2A $(\mathrm{OR}=15.21,95 \% \mathrm{CI}=4.54$ -
50.96, $\mathrm{p}<0.001)$ and DPYD*9B $(\mathrm{OR}=9.10,95 \% \mathrm{CI}=3.43-$ $24.10, \mathrm{p}<0.001)$ [37].

DPYD*13 has a frequency of $0-2 \%$ in Caucasian population [38]. Based on a meta-analysis the risk of hematological and gastrointestinal toxicities is increased up to almost 10 , respectively 6 times in patients with this variant. A heterozygous genotype is expected to result in a $40-50 \%$ decrease in DPD activity [38] (Table I).

\section{Discussion}

The main benefit in determining a genetic deficiency would be to avoid toxicity and prevent the most severe and fatal effects of toxicity by using alternative drugs or reduce fluoropyrimidine doses. In patients who need treatment with fluoropyrimidine, DPD status should be determined before the start of the treatment to prevent the potential toxicity. At this moment, clinical validity is well known for four variants (DPYD*2A, DPYD*9B, DPYD*13, and c.1236G $>$ A/Haplotype B3), but there are no official recommendations for genotyping before the treatment and also to adjust the dose based on these findings [40]. Also others characteristics such as age, gender, hepatic and renal function may increase the toxicity risk [38].

In clinical practice there are four phenotyping methods available, enzyme activity in PBM, endogenous uracil levels, $2-{ }^{13} \mathrm{C}$-uracil breath test and uracil dose test. Enzyme activity in PBM is gold-standard but is not easily implementable in clinical practice. Pretreatment uracil serum is a well feasible method in clinical practice and seems to be more predictive of fluoropyrimidine toxicity than dihydrouracil/uracil ratio. Based on the study of Meulendijks et al. it may be assumed that patients with a concentration of pretreatment serum uracil $>16 \mathrm{ngml}^{-1}$ are at increased risk of developing severe and fatal toxicity related to the treatment with fluoropyrimidine [33]. Therefore before any DPD phenotype-guided dosing can be implemented in clinical practice, further prospective studies are required and established the appropriate cut-off values. 
The most recommended strategies to increase the sensitivity of an upfront test is a DPYD genotyping and DPD phenotyping combination approach [33-34].

Even now when we know some of DPYD variant that can increase the risk of severe toxicity due to treatment with fluoropyrimidines, a negative test for these variants does not guarantee that a patient is DPD proficient. A screening only by genotyping has a low sensitivity to identify patients at risk for toxicity, and it is estimated that only a half of subjects with DPD deficiency can be identified by genotyping for the four variants (DPYD*2A, DPYD*9B, DPYD*13, and c.1236G >A/ Haplotype B3) [34-38].

The French National Network of Pharmacogenetics recommend that if genotyping has found a partial deficiency for DPYD*2A, DPYD*9B or DPYD*13 variants, the dose should be reduced by $50 \%$ for the first cycle of chemotherapy [40]. If a complete deficiency is found by genotyping, fluoropyrimidines are contraindicated and another therapy should be administrated. The Dutch Pharmacogenetics Working Group recommend using an alternative drug for homozygous patients and for heterozygous patients a reduced dose or alternative drug [39].

If the deficiency is demonstrated by phenotyping methods, the dose can be reduced in correlation with the degree of deficiency, by $25 \%$ to $75 \%$. Also, if tolerance is observed after the first cycle of chemotherapy, the dose can be increased [40].

At present, despite all studies linking DPD deficiency to fluoropyrimidine related toxicity, pharmacogenetic testing for DPYD PNS is not described in the drug label. DPD deficiency is suggested in the summary of product characteristics of Capecitabine, in both the European Union and Unites States of America, but pre-testing is not recommended.

Even if the pre-testing is not recommended due to the small incidence of the DPD deficiency, in December 2015 the Food and Drug Administration (FDA) approved uridine triacetate (Vistogard) for use in emergency cases of overdose or life-threating adverse reactions in adult and pediatric patients who undergo chemotherapy based of 5-fluorouracil or Capecitabine. Uridine triacetate is the first and the only chemotherapy antidote $[41,42]$.

\section{Conclusion}

Fluoropyrimidine-based chemotherapy is still the first line treatment in many types of cancers even in our days when targeted therapy and immunotherapy is increasingly used. The importance of chosing the right patient who will benefit the most from treatment is an important public health issue with implications not only for the patient's life, but also for the health economy. By providing the right treatment for each patient according to their own personal features we will be able to increase the effectiveness of our treatments, minimize the side effects and thus control the progression of the disease.

Despite all this data, more studies are needed, several new variants allelic to be searched, many other enzymes which can be investigated in order to diagnose those cases that are negative. Also, a phenotypic approach could be easier implemented in general practice and perhaps the time has come for one of these methods to become routine practice.

\section{References}

1. Meulendijks D, Cats A, Beijnen JH, Schellens JH. Improving safety of fluoropyrimidine chemotherapy by individualizing treatment based on dihydropyrimidine dehydrogenase activity - Ready for clinical practice? Cancer Treat Rev. 2016;50:23-34.

2. Quaranta S, Thomas F. Pharmacogenetics of anticancer drugs: State of the art and implementation recommendations of the French National Network of Pharmacogenetics. Therapie. 2017;72:205-215.

3. Mattison LK, Johnson MR, Diasio RB. A comparative analysis of translated dihydropyrimidine dehydrogenase cDNA; conservation of functional domains and relevance to genetic polymorphisms. Pharmacogenetics. 2002; 12:133144.

4. Caudle KE, Thorn CF, Klein TE, Swen JJ, McLeod HL, Diasio RB, et al. Clinical Pharmacogenetics Implementation Consortium guidelines for dihydropyrimidine dehydrogenase genotype and fluoropyrimidine dosing. Clin Pharmacol Ther. 2013;96:640-645.

5. Diasio RB, Beavers TL, Carpenter JT. Familial deficiency of dihydropyrimidine dehydrogenase. Biochemical basis for familial pyrimidinemia and severe 5-fluorouracil-induced toxicity. J Clin Invest. 1988;81:47-51.

6. Boisdron-Celle M, Remaud G, Traore S, Poirier AL, Gamelin L, Morel A, et al. 5-Fluorouracil-related severe toxicity: a comparison of different methods for the pretherapeutic detection of dihydropyrimidine dehydrogenase deficiency. Cancer Lett. 2007:249:271-282.

7. van Kuilenburg AB. Dihydropyrimidine dehydrogenase and the efficacy and toxicity of 5-fluorouracil. Eur J Cancer. 2004;40:939-950.

8. Boisdron-Celle M, Capitain O, Faroux R, Borg C, Metges JP, Galais MP, et al. Prevention of 5-fluorouracil-induced early severe toxicity by pre-therapeutic dihydropyrimidine dehydrogenase deficiency screening: Assessment of a multiparametric approach. Semin Oncol. 2017;44:13-23.

9. Lee AM, Shi Q, Pavey E, Alberts SR, Sargent DJ, Sinicrope FA, et al. DPYD variants as predictors of 5-fluorouracil toxicity in adjuvant colon cancer treatment (NCCTG N0147). J Natl Cancer Inst. 2014;106. pii: dju298. doi: 10.1093/jnci/dju298.

10. Chazal M, Etienne MC, Renée N, Bourgeon A, Richelme H, Milano G. Link between dihyropyrimidine dehydrogenase activity in peripheral blood mononuclear cells and liver. 
Clin Cancer Res. 1996;2:507-510.

11. Johnson MR, Diasio RB. Importance of dihydropyrimidine dehydrogenase (DPD) deficiency in patients exhibiting toxicity following treatment with 5-fluorouracil. Adv Enzyme Regul. 2011;41:151-157.

12. Van Kuilenburg AB, Meinsma R, Zoetekouw L, Van Gennip AH. Increased risk of grade IV neutropenia after administration of 5-fluorouracil due to a dihydropyrimidine dehydrogenase deficiency: high prevalence of the IVS14+1g>a mutation. Int J Cancer. 2002;101:253-258.

13. van Kuilenburg AB, Haasjes J, Richel DJ, Zoetekouw L, van Lenthe $H$, De Abreu RA, et al. Clinical implications of dihydropyrimidine dehydrogenase (DPD) deficiency in patients with severe 5-fluorouracil-associated toxicity: identification of new mutations in the DPD gene. Clin Cancer Res. 2000;6:4705-4712.

14. Milano G, Etienne MC, Pierrefite V, Barberi-Heyob M, Deporte-Fety R, Renée N. Dihydropyrimidine dehydrogenase deficency and fluorouracil-related toxicity. Br J Cancer. 1999;79:627-630.

15. Kristensen MH, Pedersen P, Mejer J. The value of dihydrouracil/uracil plasma ratios in predicting 5-fluorouracil-related toxicity in colorectal cancer patients. J Int Med Res. 2010;38:1313-1323.

16. Mattison LK, Ezzeldin H, Carpenter M, Modak A, Johson MR, Diasio RB. Rapid identification of dihydropyrimidine dehydrogenase deficiency by using a novel 2-13C-uracil breath test. Clin Cancer Res. 2004;10:2652-2658.

17. Cunha-Junior GF, De Marco L, Bastos-Rodrigues L, Bolina MB, Martins FL, Pianetti GA, et al. (13)C-uracil breath test to predict 5-fluorouracil toxicity in gastrointestinal cancer patients. Cancer Chemother Pharmacol. 2013;72:12731282.

18. Thomas HR, Ezzeldin HH, Guarcello V, Mattison LK, Fridley BL, Diasio RB. Genetic regulation of betaureidopropionase and its possible implication in altered uracil catabolism. Pharmacogenet Genomics. 2008;18:2535 .

19. Thomas HR, Ezzeldin HH, Guarcello V, Mattison LK, Fridley BL, Diasio RB. Genetic regulation of dihydropyrimidinase and its possible implication in altered uracil catabolism. Pharmacogenet Genomics. 2007;17:973-987.

20. van Staveren MC, Theeuwes-Oonk B, Guchelaar HJ, van Kuilenburg AB, Maring JG. Pharmacokinetics of orally administered uracil in healthy volunteers and in DPDdeficient patients, a possible tool for screening of DPD deficiency. Cancer Chemother Pharmacol. 2011;68:16111617.

21. Thorn CF, Marsh S, Carrillo MW, McLeod HL, Klein TE, Altman RB. PharmGKB summary: fluoropyrimidine pathways. Pharmacogenet Genomics. 2011;21:237-242.

22. Sobrero AF, Aschele C, Bertino JR. Fluorouracil in colorectal cancer--a tale of two drugs: implications for biochemical modulation. J Clin Oncol. 1997;15:368-381.

23. Carlsson G, Odin E, Gustavsson B, Wettergren Y. Pretherapeutic uracil and dihydrouracil levels in saliva of colorectal cancer patients are associated with toxicity during adjuvant 5-fluorouracil-based chemotherapy. Cancer Chemother Pharmacol. 2014;74:757-763.

24. Wettergren Y, Carlsson G, Odin E, Gustavsson B. Pretherapeutic uracil and dihydrouracil levels of colorectal cancer patients are associated with sex and toxic side effects during adjuvant 5-fluorouracil-based chemotherapy. Cancer. 2011;118:2935-2943.

25. Hayashi K, Kidouchi K, Sumi S, Mizokami M, Orito E, Kumada K, et al. Possible prediction of adverse reactions to pyrimidine chemotherapy from urinary pyrimidine levels and a case of asymptomatic adult dihydropyrimidinuria. Clin Cancer Res. 1996;2:1937-1941.

26. Meulendijks D, Henricks LM, Jacobs BAW, Aliev A, Deenen MJ, de Vries N, et al. Pretreatment serum uracil concentration as a predictor of severe and fatal fluoropyrimidine-associated toxicity. Br J Cancer. 2017;116:1415-1424.

27. Longley DB, Harkin P, Johnston PG. 5-fluorouracil: mechanism of action and clinical strategies. Nat Rev Cancer. 2003;3:330-338.

28. Okuda H, Nishiyama T, Ogura K, Nagayama S, Ikeda K, Yamaguchi S, et al. Lethal drug interactions of sorivudine, a new antiviral drug, with oral 5-fluorouracil prodrugs. Drug Metab Dispos. 1997;25:270-273.

29. van Kuilenburg AB, Muller EW, Haasjes J, Meinsma R, Zoetekouw L, Waterham HR, et al. Lethal outcome of a patient with a complete dihydropyrimidine dehydrogenase (DPD) deficiency after administration of 5-fluorouracil: frequency of the common IVS14+1G $>$ A mutation causing DPD deficiency. Clin Cancer Res. 2001;7:1149-1153.

30. Rosmarin D, Palles C, Church D, Domingo E, Jones A, Johnstone E, et al. Genetic markers of toxicity from capecitabine and other fluorouracil-based regimens: investigation in the QUASAR2 study, systematic review, and meta-analysis. J Clin Oncol. 2014;32:1031-1039.

31. Meulendijks D, Henricks LM, Sonke GS, Deenen MJ, Froehlich TK, Amstutz U, et al. Clinical relevance of DPYD variants c. $1679 \mathrm{~T}>\mathrm{G}$, c. $1236 \mathrm{G}>\mathrm{A} / \mathrm{HapB} 3$, and c. $1601 \mathrm{G}>\mathrm{A}$ as predictors of severe fluoropyrimidine-associated toxicity: a systematic review and meta-analysis of individual patient data. Lancet Oncol. 2015;16:1639-1650.

32. Lam SW, Guchelaar HJ, Boven E. The role of pharmacogenetics in capecitabine efficacy and toxicity. Cancer Treat Rev. 2016;50:9-22.

33. Mercier C, Ciccolini J. Profiling dihydropyrimidine dehydrogenase deficiency in patients with cancer undergoing 5-fluorouracil/capecitabine therapy. Clin Colorectal Cancer. 2006;6:288-296.

34. Terrazzino S, Cargnin S, Del Re M, Danesi R, Canonico PL, Genazzani AA. DPYD IVS14+1G $>$ A and 2846A $>$ T genotyping for the prediction of severe fluoropyrimidinerelated toxicity: a meta-analysis. Pharmacogenomics. 2013;14:1255-1272.

35. Offer SM, Fossum CC, Wegner NJ, Stuflesser AJ, Butterfield GL, Diasio RB. Comparative functional analysis of DPYD variants of potential clinical relevance to dihydropyrimidine dehydrogenase activity. Cancer Res. 2014;74:2545-2554.

36. Henricks LM, Opdam FL, Beijnen JH, Cats A, Schellens 
JHM. DPYD genotype-guided dose individualization to improve patient safety of fluoropyrimidine therapy: call for a drug label update. Ann Oncol. 2017;28:2915-2922.

37. Ruzzo A, Graziano F, Galli F, Galli F, Rulli E, Lonardi S, et al. Dihydropyrimidine dehydrogenase pharmacogenetics for predicting fluoropyrimidine-related toxicity in the randomised, phase III adjuvant TOSCA trial in high-risk colon cancer patients. Br J Cancer. 2017;117:1269-1277.

38. Matáková T, Halašová E, Škovierová H, Dzian A, Dobrota D, Škereňová M. DPYD genotype and haplotype analysis and colorectal cancer susceptibility in a case-control study from Slovakia. Gen Physiol Biophys. 2017;36:557-563.

39. Bank PCD, Caudle KE, Swen JJ, Gammal RS, WhirlCarrillo M, Klein TE, et al. Comparison of the Guidelines of the Clinical Pharmacogenetics Implementation Consortium and the Dutch Pharmacogenetics Working Group. Clin Pharmacol Ther. 2018;103:599-618.
40. Loriot MA, Ciccolini J, Thomas F, Barin-Le-Guellec C, Royer B, Milano G, et al. Dihydropyrimidine déhydrogenase (DPD) deficiency screening and securing of fluoropyrimidine-based chemotherapies: Update and recommendations of the French GPCO-Unicancer and RNPGx networks. Bull Cancer. 2018;105:397-407.

41. Ison G, Beaver JA, McGuinn WD Jr, Palmby TR, Dinin J, Charlab R, et al. FDA Approval: Uridine Triacetate for the Treatment of Patients Following Fluorouracil or Capecitabine Overdose or Exhibiting Early-Onset Severe Toxicities Following Administration of These Drugs. Clin Cancer Res. 2016;22:4545-4549.

42. Ma WW, Saif MW, El-Rayes BF, Fakih MG, Cartwright TH, Posey JA, et al. Emergency use of uridine triacetate for the prevention and treatment of life-threatening 5-fluorouracil and capecitabine toxicity. Cancer. 2017;123:345-356. 